\title{
High Resolution Transmission Electron Microscopy Study of Montmorillonite Subjected to Rapid Compression
}

\author{
Yanyan Zhang ${ }^{1}$, Maobin $\mathrm{Wei}^{2}$, Yanzhang $\mathrm{Ma}^{1,3}$ and Jharna Chaudhuri ${ }^{3}$ \\ 1. Center for High Pressure Science and Technology Advanced Research, 2699 Qianjin Street, \\ Changchun, China, \\ 2. Institute of Condensed State Physics, Key Laboratory of Functional Materials Physics and Chemistry \\ of the Ministry of Education, Jilin Normal University, Siping, China, \\ 3. Department of Mechanical Engineering, Texas Technology University, 2703 7th Street, Lubbock, \\ USA, TX
}

Many of the common clays are aluminosilicates, which have a sheet-like (layered) structure, and consist of silica $\mathrm{SiO}_{4}$ tetrahedra bonded to alumina $\mathrm{AlO}_{6}$ or $\mathrm{AlOH}$ octahedra in a variety of ways. A 2:1 ratio of the tetrahedra to the octahedra results in smectite clays, the most common of which is montmorillonite (MMT). Other metals such as magnesium may replace the aluminum in the crystal structure. In this tetrahedra-octahedra-tetrahedra (TOT) structure the central octahedral alumina sheet is sandwiched between two tetrahedral silica sheets. The clay layers allow delocalization of negative charges. These negative charges are then neutralized by cations such as $\mathrm{H}^{+}, \mathrm{Na}^{+}, \mathrm{K}^{+}, \mathrm{Mg}^{2+}$ or $\mathrm{Ca}^{2+}$ situated in between the charged layers. Because of their high hydrophilic nature, water molecules generally position between the clay layers as well $[1,2]$.

Rapid compression [3] studies have a special place in the study of minerals in earth's interior since the entire pressure and temperature ranges can be obtained in the laboratory. Characterization of rapid compressed minerals could provide important information on nature of shock formation mechanism [4].

Electron diffraction provides access to structural information of crystalline material down to the nanoscale. High-resolution transmission electron microscopy (HRTEM) allows for direct imaging of the atomic structure of the sample and interplanar spacings. In this research, electron diffraction and high resolution transmission electron microscopy (HRTEM) investigation were performed on $\mathrm{Na}^{+}-$ montmorillonite. Selected area diffraction pattern (SADP) method was used to take the electron diffraction. Energy dispersive spectroscopic (EDS) was performed to obtain the elements present in the sample. A JEOL $2100 \mathrm{TEM}$ at $200 \mathrm{KV}$ with $0.2 \mathrm{~nm}$ resolution was used. Four different samples were studied as follows: original montmorillonite, one sample under rapid compression, one sample annealed in Ar gas at $1000^{\circ} \mathrm{C}$, and one annealed sample after rapid compression. The aim was to assess the interplanar spacings of the samples subjected to rapid compression.

Figure 1 (a) shows the TEM image of the original montmorillonite sample, where the layer structure is clearly visible. The elemental analysis of the original sample indicates that the elements present are Al, $\mathrm{Si}, \mathrm{Mg}, \mathrm{Fe}, \mathrm{Na}, \mathrm{Ca}$ and $\mathrm{O}$ (Figure 1 (b)). The electron diffraction pattern (EDP) from the original sample clearly shows that the samples are single crystalline (Figure 1 (c)). One representative lattice imaging from the original sample is shown in Figures 1(d) where the corresponding interplanar spacing is indicated. Table 1 shows the calculated and experimental interplanar spacings. The calculated interplanar spacings were obtained using the lattice parameters from Reference [2] as $a=5.18 \AA, b=$ $8.98 \AA, c=10.08 \AA$ and $\beta=100.2^{\circ}$ with a monoclinic unit cell and a space group $\mathrm{C} 2 / \mathrm{m}$. The interplanar spacings were calculated assuming dry aluminosilicate layers (column 4 in Table 1) and one layer of 
water molecule absorbed in the [001] direction and hence changing the $c$ lattice parameter to $12.83 \AA$ (column 5 in Table 1) [5]. Columns 6 to 9 in Table 1 are the interplanar spacings obtained from HRTEM lattice imaging. It can be clearly seen that the original montmorillonite sample had absorbed water layer. The water layer caused a marked increase in the $c$ lattice parameter. In fact $16 \AA$ of $\mathrm{d}_{001}$ value in original montmorillonite (table 1) might indicate presence of two water layers within the unit cell. In contrast, the water squeezed out when the MMT was subjected to compression and annealing. It has been mentioned in the literature that with increased hydration montmorillonite attains most energetically advantageous and least stressed crystal structure while the structure disintegrates with dehydration [5].

\section{References:}

[1] JM Cowley and A Goswami, Acta Cryst. 14 (1961), p. 1071.

[2] SI Tsipursky and VA Drits, Clay Minerals 19 (1984), p. 177.

[3] LY Chen, et al., Mater. Res. Express 1 (2014), p. 025707.

[4] TJ Ahrens, Methods of Experimental Physics 24A (1987), p. 185.

[5] MV Eirish and LI Trety’ikova, Clay Minerals 8 (1970), p. 255.

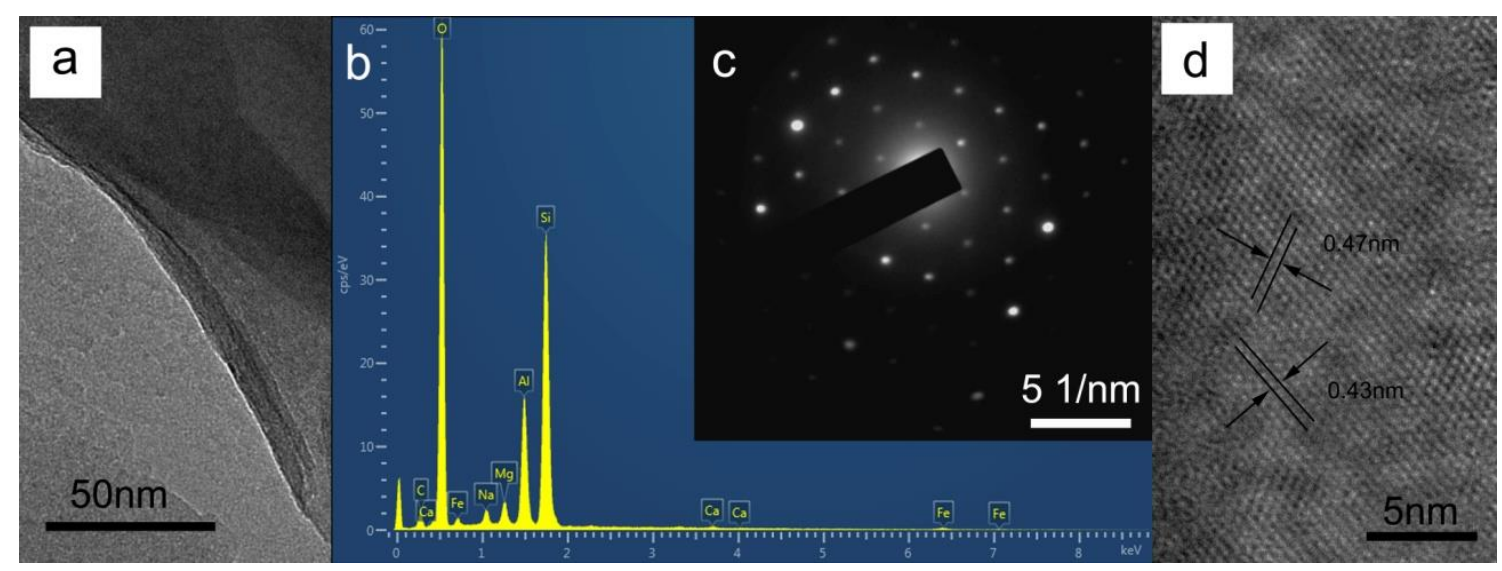

Figure 1. (a) Morphology, (b) EDS spectra, (c) EDP and (d) HRTEM lattice imaging of the of original montmorillonite sample.

\begin{tabular}{|c|c|c|c|c|c|c|c|c|}
\hline \multirow[b]{2}{*}{ h } & \multirow[b]{2}{*}{ k } & & \multicolumn{2}{|c|}{ Calculated d $(\dot{\AA})$} & \multicolumn{4}{|c|}{ Measured d from HRTEM Lattice Imaging $(\overline{\mathrm{A}})$} \\
\hline & & & Dry & Hydrated & \begin{tabular}{|l} 
Original \\
sample
\end{tabular} & $\begin{array}{l}\text { Compressed } \\
\text { sample }\end{array}$ & Annealed sample & \begin{tabular}{|l|} 
Annealed and \\
Compressed sample
\end{tabular} \\
\hline 0 & 0 & 1 & 9.92 & 12.72 & 16.0 & 10.4 & & 10.4 \\
\hline 0 & 1 & 1 & 6.65 & 7.33 & & & & $7.4,6.3,5.6$ \\
\hline 0 & 0 & 2 & 4.96 & 6.36 & 5.3 & & $4.8,4.7,4.6$ & \\
\hline 0 & 2 & 0 & 4.49 & 4.49 & 4.7 & 4.5 & & \\
\hline 0 & 2 & 1 & 4.09 & 4.23 & 4.3 & & 4.0 & \\
\hline 1 & 1 & 1 & 3.83 & 4.0 & 4.1 & & $3.9,3.7$ & 4.0 \\
\hline-1 & 1 & 2 & 3.59 & 3.93 & & 3.6 & & \\
\hline 0 & 2 & 2 & 3.32 & 3.66 & 3.5 & & & $3.4,3.6$ \\
\hline 1 & 1 & 2 & 3.08 & 3.40 & & $3.18,3.04,2.96$ & 3.0 & \\
\hline-1 & 1 & 3 & 2.87 & 3.33 & 3.3 & $2.8,2.74,2.85$ & $2.9,2.8$ & \\
\hline 0 & 2 & 3 & 2.66 & 3.08 & 2.9 & $2.66,2.64,2.70$ & & \\
\hline 1 & 1 & 3 & 2.47 & 2.85 & $2.7,2.8$ & 2.51 & & 2.5 \\
\hline 0 & 2 & 4 & 2.17 & 2.60 & & $2.2,2.17,2.28$ & & \\
\hline 1 & 1 & 4 & 2.04 & 2.41 & & 1.9 & & \\
\hline 0 & 2 & 5 & 1.81 & 2.21 & 2.2 & & & \\
\hline
\end{tabular}

Table 1. The interplanar spacings, d, calculated for MMT, dry and with one layer of water, and measured from HRTEM images. 